\title{
L’érosion De La Falaise De Sidi Bouzid (Côte Atlantique Marocaine) : Quantification Et Évolution Spatio-Temporelle
}

\author{
Soukaina Khouaja \\ Mohamed Ouadia \\ Imane Mourabiti \\ El Mehdi Irzan
}

Université Chouaib Doukkali, Faculté des Sciences,

Département de Géologie, Laboratoire de Géosciences et Techniques de

l’Environnement, El Jadida, Maroc

doi: 10.19044/esj.2016.v12n6p133 URL:http://dx.doi.org/10.19044/esj.2016.v12n6p133

\section{Abstract}

The study of the coastal cliff "F1" of Sidi Bouzid shows that it is discontinuous, high, complex and very exposed to the marine dynamics. It is subdivided into two sectors in total, consisting of five zones. A second small cliff "F2" is visible, at the foot of F1 during the storms. These two cliffs bear witness to an oscillation of sea level during the Recent Quaternary. This work shows also a clear close relationship between the quantity and the quality of the blocks, on the one hand, and the nature of the fairly limited space on which have accumulated on the other hand. At the stratigraphic level, the cliff in the Northeast sector is characterized by alternating beds of limestone and Cretaceous Marly levels with evidence of tectonic activity. In the Southwest, Quaternary is dominated by mainly continental deposits, with caliches, overcoming the Cretaceous limestones. The block settings are variable and influenced by the height of the cliff. The degree of fragmentation of blocks during their fall affects their shape: elongated (zone V) because little fragile joints and resistance as well as thick banks of limestone or less elongated because hardness of the platform and fragile joints (zone I). Reflecting that blocks inherit, their form of the morphology, structure and lithological nature of the formations that make up. Quantitatively, the number, density and the volume of blocks are variable and proportional but important (I and $\mathrm{V}$ zones) of active erosion due to exposure of the cliff to the marine dynamics. However, the number and low density with the high volume of blocks (zone III) reflect indurated and lithological nature (caliches) but less rich in joints of Quaternary deposits. 
On the indices of the blocks, they show little variation but a slight peculiarity of zone $\mathrm{V}$ (extended blocks). The layout of blocks shows a difference between blocks of the Northeast and the Southwest sector due to the different orientation of the cliffs but also the turn and the onslaught of the waves in zone III. This study also shows a clear decrease in the cliff and the risk that it is requiring for its protection.

Keywords: Moroccan Atlantic coast, Sidi Bouzid, Cliff, erosion, landslides, quantification, spatio-temporal evolution, marine dynamic, risk

\section{Résumé}

L'étude de la falaise côtière «F1 » de Sidi Bouzid montre qu'elle est discontinue, haute, complexe et très exposée à la dynamique marine. Elle est subdivisée en deux secteurs composés, au total, de cinq zones. Une deuxième petite falaise «F2 » n'est visible, au pied de F1 que lors des tempêtes. Ces deux falaises témoignent d'une oscillation du niveau marin lors du Quaternaire récent. Ce travail, montre également une nette relation étroite entre la quantité et la qualité des blocs, d'une part, et la nature de l'espace assez restreint sur lequel se sont accumulés d'autre part. Sur le plan stratigraphique, la falaise au secteur nord-est est caractérisée par une alternance de bancs de calcaires et de niveaux marneux crétacés riches en indice d'activité tectonique. Quant au Sud-Ouest, se sont les dépôts quaternaires essentiellement continentaux, très encroûtés, surmontant les calcaires crétacés, qui dominent. Les paramètres des blocs sont variables et influencés par la hauteur de la falaise. Le degré de la fragmentation des blocs lors de leur chute influe leur forme : allongée (zone V) car diaclases peu fragilisées et résistante ainsi que l'épaisseur importante des bancs de calcaires ou moins allongée car dureté de la plateforme et diaclases fragilisées (zone I). Ce qui reflète que les blocs héritent, leur forme de la morphologie, de la structure et de la nature lithologique des formations qui la composent. Quantitativement, le nombre, la densité et le volume des blocs sont variables et proportionnels mais importants (zones I et V) témoignant d'une érosion active due à une exposition de la falaise à la dynamique marine. Cependant, le nombre et la densité faibles avec le volume fort des blocs (zone III) traduisent la nature lithologique encroûtée et indurée mais moins riche en diaclases des dépôts quaternaires. Concernant les indices des blocs, ils montrent peu de variations mais une légère particularité de la zone $\mathrm{V}$ (blocs allongés). La disposition des blocs montre une différence entre les blocs du secteur nord-est et ceux du secteur sud-ouest à cause de l'orientation différente des falaises mais aussi du virage et du déferlement des vagues dans la zone III. Cette étude prouve également un net recul de la 
falaise et le risque qu’elle présente. Elle nécessite une intervention pour sa protection.

Mots-clés : Côte atlantique marocaine, Sidi Bouzid, falaise, érosion, éboulements, quantification, évolution spatio-temporelle, dynamique marine, risque

\section{Introduction}

La baie de Sidi Bouzid, faisant partie de la côte atlantique marocaine au Sud-Ouest d'El Jadida, est connue par sa belle plage sableuse renommée à l'échelle nationale. Cette dernière est interrompue par une plage rocheuse à falaise haute et vive qui fait l'objet de cette étude. Sur le plan socioéconomique, plusieurs activités y sont développées dont le tourisme, la pêche et l'exploitation des algues rouges.

A notre connaissance, jusqu’à nos jours, aucune étude s’intéressant à l'érosion de cette falaise n'est entreprise à l'exception de certaines études purement stratigraphiques. Le but de ce travail est d'essayer, pour la première fois, de faire une analyse, quantitative et qualitative de l'érosion de cette falaise et de ses éboulements. Ce travail permet également de mettre en relief les divers mécanismes, en particulier la dynamique marine, responsables de cette érosion, du recul de la falaise ainsi que de leur évolution spatio-temporelle.

Enfin, la menace et le risque que cette érosion engendre pour la falaise et les infrastructures avoisinantes sont aussi discutés dans cette étude.

\section{Situation Géographique}

La région étudiée fait partie de la côte atlantique marocaine, elle est limitée au Nord-Est par la ville d'El Jadida et au Sud-Ouest par la commune de Moulay Abdellah. Ses coordonnées Lambert sont : 33¹3'02.55'’Nord / 8³3'51.41'’Ouest et 33¹3'12.31'’Nord / 8³3'38.87'’Ouest (Fig. 1).

Le secteur d'étude, objet de ce travail, est composé d'une importante falaise façonnée, essentiellement, dans des calcaires crétacés pour la partie nord-est surmonté par des dépôts quaternaires dans la partie sud-ouest. Elle limite la plage de Sidi Bouzid au Sud-Ouest mais elle est discontinue étant donnée qu'elle est interrompue par une petite plage 'Beau séjour' ou « crique de Sidi Bouzid » (Gigout M., 1951; Aboumaria K., 1993 et Ouadia M., 1998).Ce secteur sépare, également, les deux baies à plages sableuses et plus fréquentées lors des périodes estivales : la plus grande Sidi Bouzid au Nord-Est et celle de Sidi El Abed au Sud-Ouest. La falaise «F1 » qui nous intéresse dans ce travail est vive et sous l'action d'une importante érosion marine. Les amas de dépôts accumulés à son pied sont formés de blocs de taille et de forme diverses. Des mesures de ces blocs ont été effectuées. 


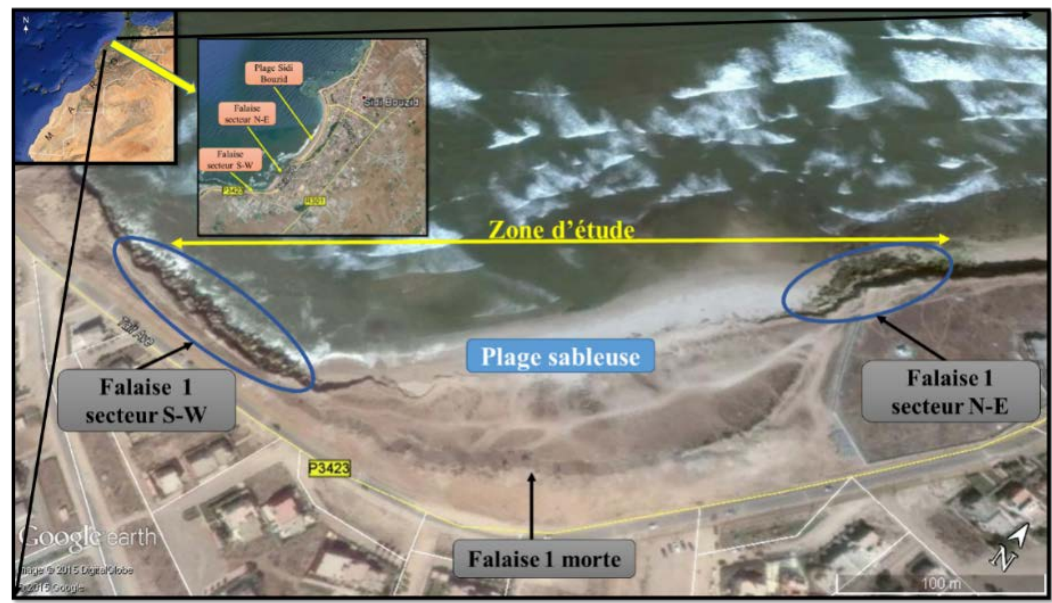

Figure 1: Situation géographique de la falaise de Sidi Bouzid (photos Google Earth).

\section{Matériel et méthodes}

Afin de comprendre les différents processus de l'érosion de la falaise de Sidi Bouzid on a fait appel à des mensurations relatives à la fois à la falaise et aux blocs résultant de son érosion. Cette méthode est celle appliquée, normalement, aux galets (Tricart et al., 1955). Mais dans notre cas, on se limite seulement aux paramètres des blocs et quelques indices propres à ces derniers du fait que, contrairement aux galets, c'est la gravité qui l'emporte sur les autres agents de l'érosion. L'étude des blocs est à la fois quantitative et qualitative. Elle intéresse, surtout, la forme, la taille et la quantité des blocs accumulés au pied de la falaise.

Les paramètres mesurés pour chaque bloc sont: Ls : longueur de la face supérieure; Li : Longueur de la face inférieure; ls : largeur de la face supérieure; li : Largeur de la face inférieure; E : épaisseur et Di : diagonale. Les valeurs utilisées pour les graphiques sont des moyennes. Pour comprendre et expliquer la disposition des blocs, des mesures du degré de leur inclinaison et de la direction de leur axe sont prises.

Concernant la falaise, les mesures faites sont celles de la hauteur, de la pente et des distances séparant son pied du rivage, du niveau de la haute et de la basse marée.

Sur le terrain, le matériel utilisé, comprend en particulier: le mètre, la boussole, le GPS, le niveleur, les cartes topographiques et géologiques en plus des photographies aériennes et des photos de Google Earth. Une fois les paramètres mesurés, des calculs de certains indices ont été faits. Ensuite, des graphiques ont été établis, commentés et interprétés. 


\section{Résultats}

\section{La falaise}

\section{Description}

La falaise côtière de Sidi Bouzid est haute et discontinue, étant donné qu'elle est interrompue par une petite plage sableuse la séparant en deux parties (Sud-Ouest et Nord-Est) présentant chacune un petit cap (Fig. 2 et 3). Latéralement, sa forme et sa hauteur varient peu. Elle est étendue sur une distance d'environ $390 \mathrm{~m}$ et d'une hauteur qui peut atteindre $10 \mathrm{~m}$. Elle est très exposée à l'action de la dynamique marine et sa pente est irrégulière. Par endroit, en particulier dans la falaise nord, une deuxième falaise (F2) est présente qui peut atteindre $2 \mathrm{~m}$ de hauteur.

Figure 2: Subdivision de la falaise de Sidi Bouzid en trois zones (secteur S-W).

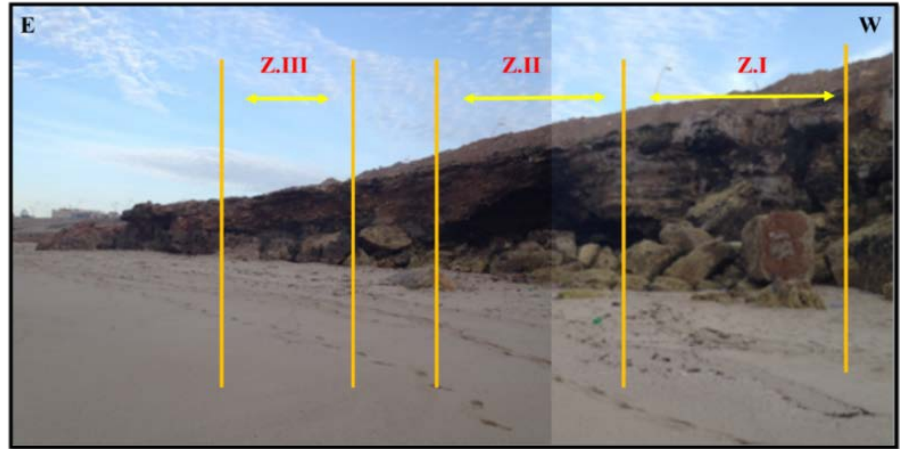

Figure 3: Subdivision de la falaise de Sidi Bouzid en 2 zones (secteur N-E).

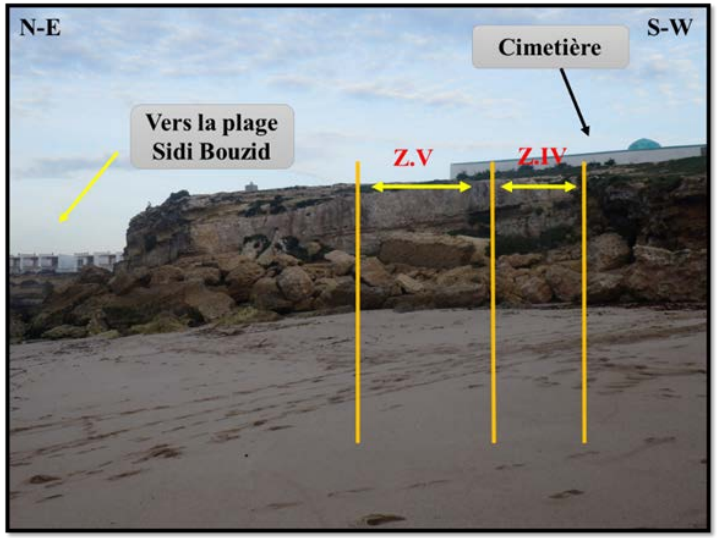

Le profil et l'espace la séparant du niveau de la basse marée est très restreint et variable, ils abritent de nombreux blocs de forme et de taille différentes.

A la surface, la végétation est peu développée pour la falaise 1 au Nord-Est et absente pour la falaise au Sud-Ouest.

Aux bords de la région étudiée, il s'agit d'une falaise vive polycyclique étant donné qu'elle est façonnée par la transgression ouljienne 
puis par la transgression mélahienne. Cependant, au centre de la baie, la falaise est également vive et monocyclique car elle est façonnée uniquement par la transgression mélahienne.

La stratigraphie de la falaise au Nord est complètement différente de celle au Sud. Elle montre un plissement des formations et de nombreuses diaclases qui les affectent.Au Nord, la falaise est composée d'une alternance de bancs calcaires massifs et épais avec des niveaux moins saillants marneux. L'ensemble est d’âge crétacé, plus précisément cénomanien (Khatmi S., 1999)(Fig. 4). Au Sud, elle est constituée de bancs de calcaires marins crétacés, surmontés par des dépôts quaternaires. Ces derniers sont constitués, de bas en haut de, dépôts lumachelliques marins, de calcarénites continentales, de paléosols rouges, d'une croûte calcaire discontinue, de dépôts de pente englobant des cailloux anguleux enrobés dans un paléosol rouge remanié et enfin de niveau épais très encroûté à la surface (Fig. 5).

Figure 4: Coupe lithostratigraphique de la falaise dans le secteur N-E.
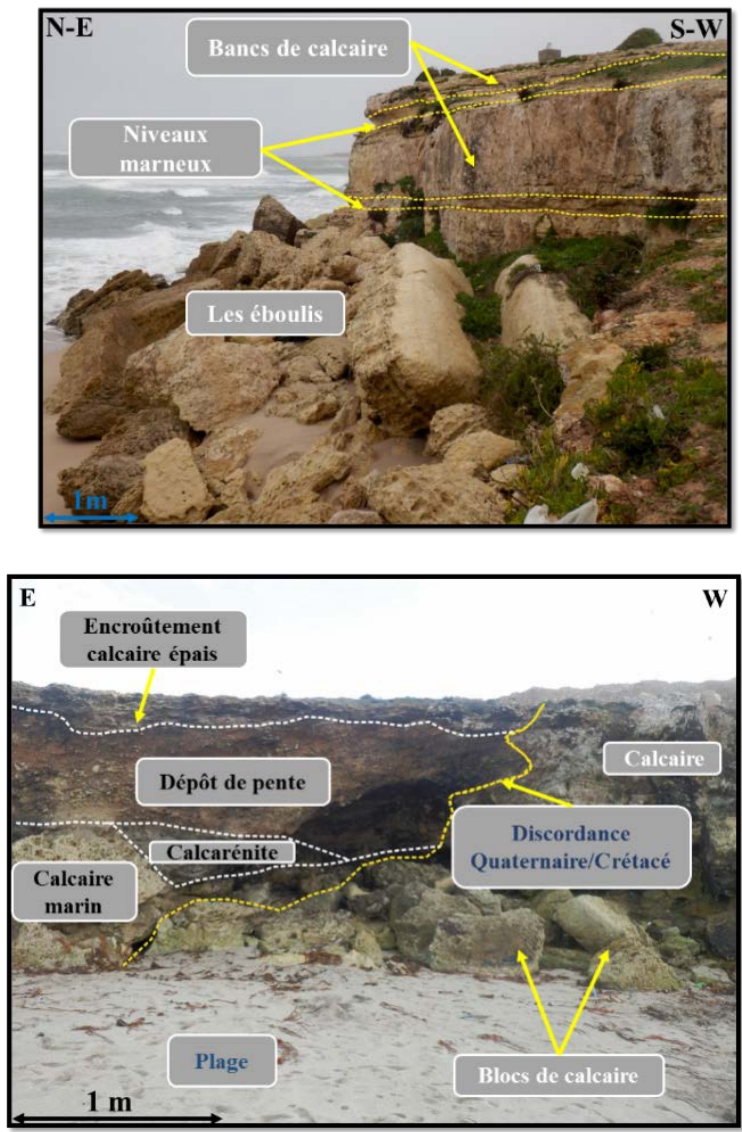

Figure5: Coupe lithostratigraphique de la falaise dans le secteur S-W (en noir les formations quaternaires et en blanc les formations crétacées). 


\section{Géomorphologie}

Sur le plan géomorphologique, la falaise 1 fait partie d'une région dont la paléogéographie est complexe. Elle est affectée par une tectonique anté-quaternaire rendant les formations crétacées inclinées vers le SudOuest, plissées et riches en diaclases et par une néotectonique ayant laissé des traces dans les formations quaternaires (Ouadia M. et al., 2015). La transgression ouljienne a pu donner naissance à la falaise 1 dite ouljienne. Cette dernière devient morte au centre de la région étudiée (Fig. 1). Mais elle est une autre fois attaquée par la transgression mélahienne au niveau des zones exposées à la mer (secteurs étudiés) et elle est devenue vive. Le plateau (sommet de la falaise) présente, dans le secteur sud-ouest, une pente relativement moyenne favorisant la mise en place de dépôts de pentes quaternaires. Cette géomorphologie a, largement, influencé l'érosion de la falaise étudiée (Akil M. 1990 et Ouadia M., 1998).

\section{Recul de la falaise}

Pour estimer l'ampleur du recul de la falaise de Sidi Bouzid, suite à son érosion, on a procédé à la comparaison des deux photos Google Earth (2001 et 2015). De cette étude ressort que l'érosion attaque la falaise de manière différente en fonction des zones (Fig. 2 et 3). Ce recul est en moyenne de l'ordre de $17,33 \mathrm{~cm} / \mathrm{an}$. Il est variable d'une zone à l'autre (Centre de la falaise : $10 \mathrm{~cm} / \mathrm{an}$; Secteur N-E : $21 \mathrm{~cm} /$ an et Secteur SW: 21cm/an) (Fig. 6). Ces chiffres témoignent que la falaise étudiée est vraiment menacée (comme d'autres régions côtières au Maroc : Aberkan M., 1996, Aberkan M. et al., 1997 et Sbai et al. 2004) et présente un risque pour la région et les infrastructures aux alentours si aucune précaution et protection ne sont entreprises.

Figure 6: Evolution du recul de la falaise de Sidi Bouzid entre 2001 et 2015 (photos Google Earth traitée).

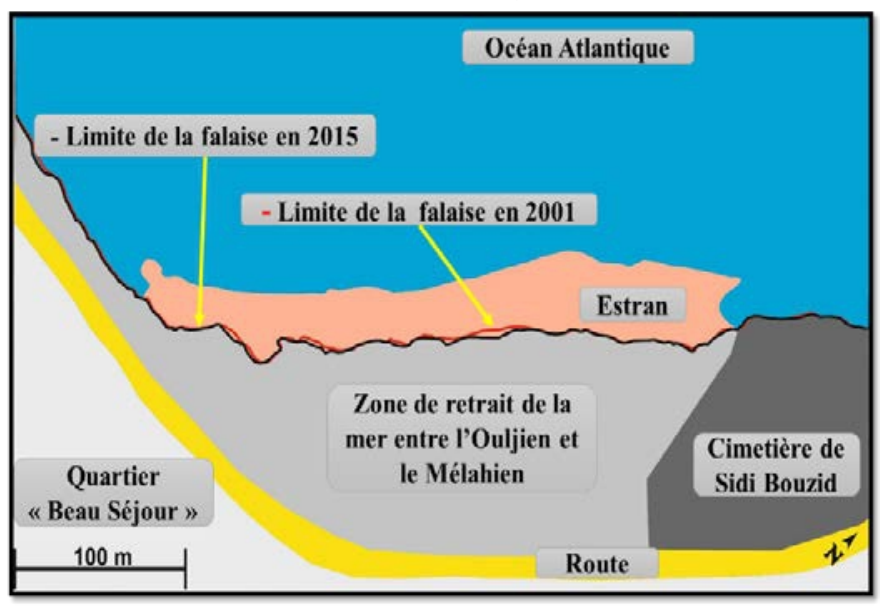




\section{Variation spatio-temporelle de la falaise}

Si le recul de la falaise est légèrement différent d'une zone à l'autre, ceci témoigne que son érosion dans le temps dépend également des variations spatiales et influe l'environnement dont la belle plage de Sidi Bouzid. En effet, chaque zone ne ressemble pas à l'autre. Par conséquent, les facteurs principaux responsables de cette érosion dont la hauteur, l'exposition et la lithologie des formations de la falaise ainsi que le degré de fragilisation des diaclases varient aussi du Nord-Est au Sud-Ouest.

\section{Les éboulements}

\section{Analyse quantitative}

Concernant les valeurs du nombre et de la densité des blocs, les plus importantes sont celles de la zone V suivie de celles de la zone IV (Fig. 7). Celles des zones I et II sont moyennes. Par contre, celles de la zone III sont les plus faibles.

A propos du volume, les valeurs les plus importantes sont celles des zones I et V suivies de celles des zones III. Les zones II et IV présentent les blocs dont le volume est faible (Fig. 8).

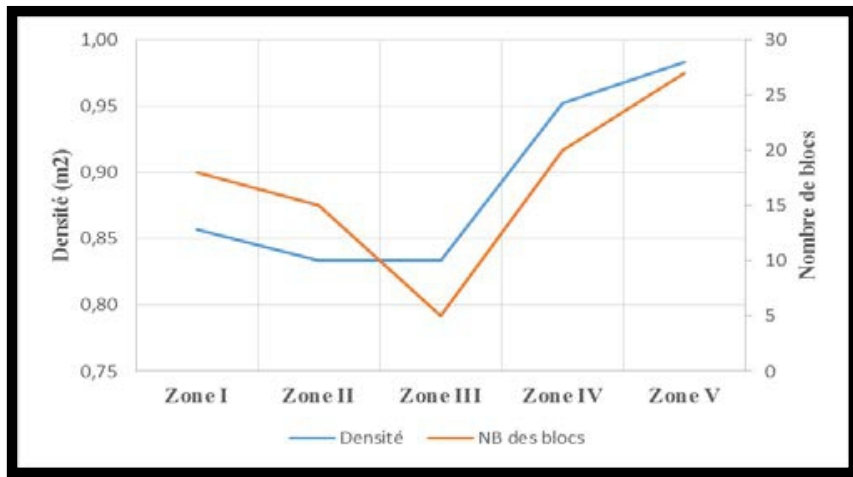

Figure 7: Evolution du nombre et de la densité des blocs.

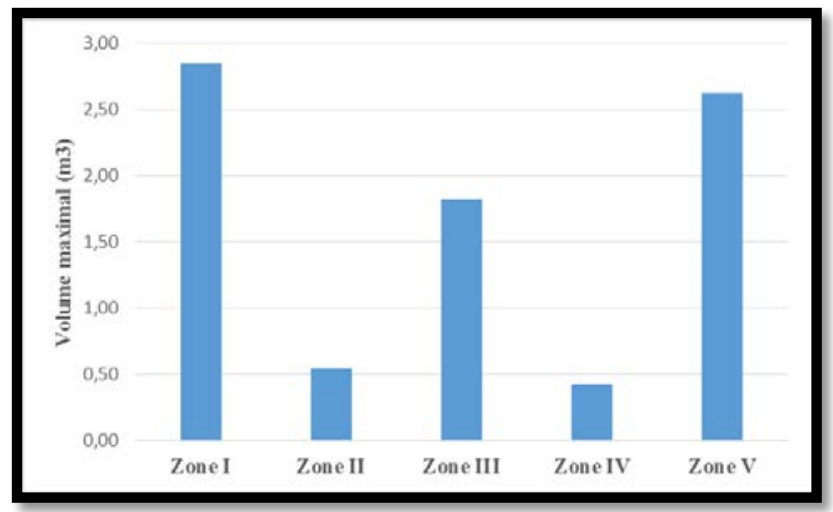

Figure 8: Evolution du volume maximal des blocs. 


\section{Analyse qualitative}

\section{Paramètres des blocs}

Les valeurs de différents paramètres des blocs sont variables. Les plus élevées sont celles de la zone V, les moyennes sont celles des zones I et III et les plus faibles sont celles des zones II et IV. La variation de ces paramètres, au sein de chaque zone, se ressemble à l'exception de la zone $\mathrm{V}$ où on note un grand écart entre la longueur $(2,06 \mathrm{~m})$ et la diagonale $(1,43 \mathrm{~m})$ des blocs,-d'une part, et la largeur (0,99 $\mathrm{m})$ et l'épaisseur (0,47 $\mathrm{m})$ de ceux-ci de l'autre part (zone III). En plus, dans la zone I, la largeur inférieure et l'épaisseur des blocs sont presque identiques (Fig. 9).

Figure 9: Evolution des paramètres des blocs.

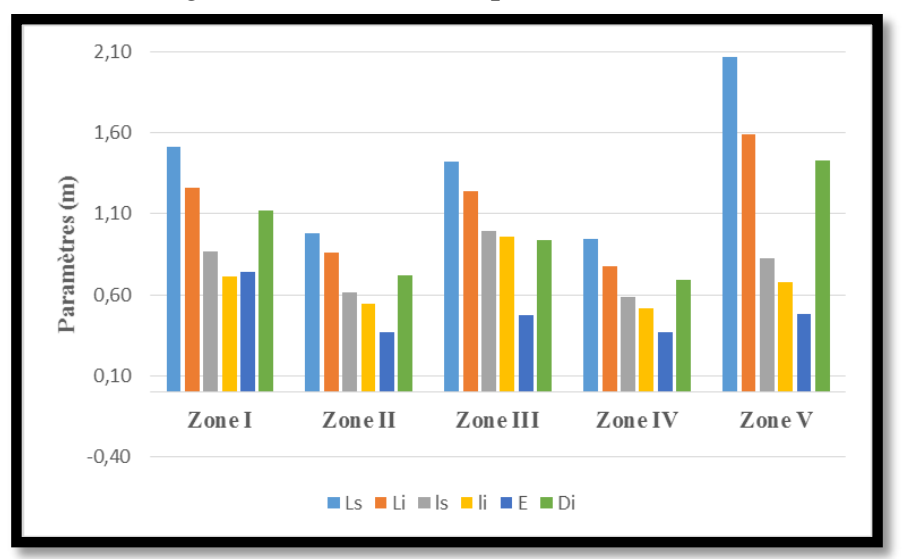

\section{Indices des blocs}

Les valeurs des indices des blocs Ls/E sont les plus élevées, suivies de celles de Ls/Di puis de celles de Ls/Li. Une ressemblance est notée entre les valeurs de l'évolution de Ls/Li et de Ls/Di. Par contre, les valeurs de Ls/E évoluent autrement. En plus, c'est dans la zone V où Ls/E est le plus élevé. Ls/Li est presque constant et ne varie presque pas dans les trois zones I, II et III. En outre, un net contraste est noté entre les valeurs de $\mathrm{Ls} / \mathrm{Li}$ et celles de Ls/Di dans la zone III (Fig. 10).

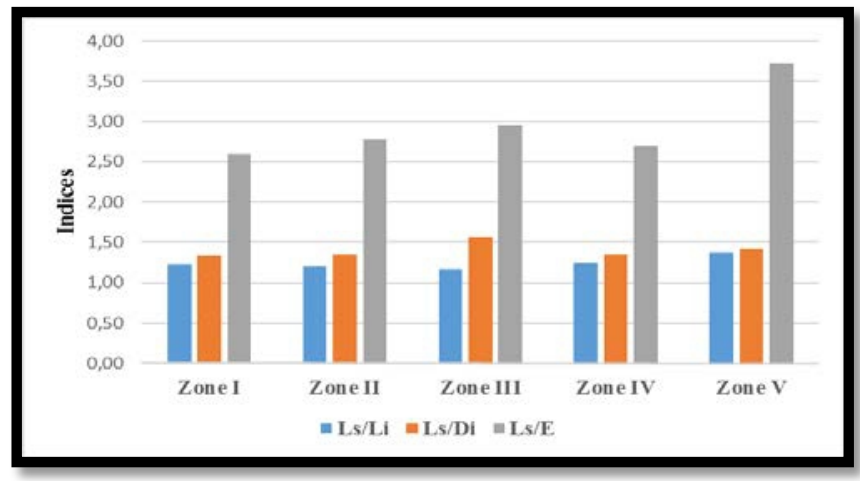

Figure 10: Evolution des indices des blocs 


\section{Disposition des blocs}

Quant à la direction de l'axe des blocs, ses valeurs varient entre $99^{\circ}$ et $49^{\circ}$. Celles de la direction de l'inclinaison des blocs entre $137^{\circ}, 40^{\prime \prime}$ ' et $70^{\circ}, 16^{\prime}$ '. Cependant, les valeurs de l'inclinaison des blocs oscillent entre $39^{\circ}, 63^{\prime \prime}$ ' et $29^{\circ}$ (Fig. 11).

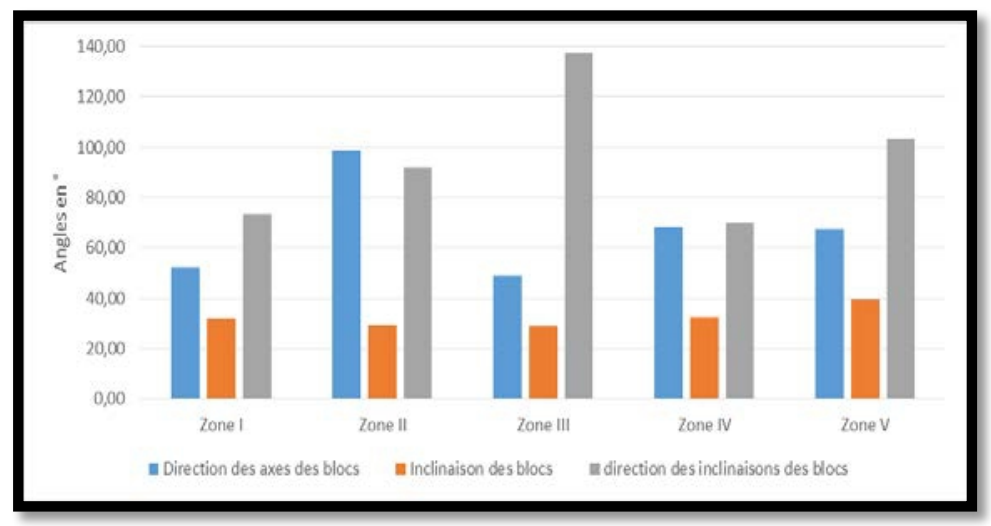

Figure 11: Evolution de la direction de l'axe d'orientation des blocs.

\section{Discussions et interprétations}

L'étude descriptive de la falaise côtière de Sidi Bouzid «Falaise 1 » montre que cette dernière est, de point de vue géomorphologique, très diversifiée. Elle est d'une étendue d'environ $390 \mathrm{~m}$ et de hauteur maximale de $10 \mathrm{~m}$. Elle est très exposée à l'action de la dynamique marine. Son interruption par une petite plage sableuse nous permet de la séparer en deux secteurs (sous forme de caps) : un au nord-est subdivisé en deux zones et un au sud-ouest subdivisé en trois zones. Seules les parties accessibles des deux secteurs, sont étudiées (Tab. 1). Si la falaise principale «F1 » est polycyclique (façonnée par des transgressions ouljienne et mélahienne), la seconde «F2 » est monocyclique (creusée uniquement lors de la transgression mélahienne) et d'environ $2 \mathrm{~m}$ de hauteur. Elle n'existe que par endroit mais affleure uniquement lors des tempêtes pour le cas du secteur nord-est, ce qui témoigne d'une nette évolution spatiale de cette falaise. Le recul calculé, non négligeable, de la falaise montre que cette dernière présente un risque et sollicite une intervention pour sa protection.

L'espace, généralement restreint, séparant le pied de la falaise et le niveau de la basse marée est en relation étroite avec la quantité et la qualité des blocs qui y sont accumulés.

Pour les deux secteurs, les formations basales (calcaires crétacés) sont plissées, inclinées et renferment de nombreuses diaclases témoignant d'une activité tectonique. Au Nord, l’hétérogénéité de la falaise s'explique par une nette alternance de bancs calcaires massifs et épais avec des niveaux marneux moins saillants. Au Sud, les calcaires marins crétacés sont 
surmontés par des dépôts quaternaires marins et continentaux encroûtés à faciès variables les permettant d'acquérir une résistance (croûte calcaire indurée : Vogt T., 1983), par endroit, vis-à-vis la dynamique marine.

\begin{tabular}{|c|c|c|c|c|c|c|c|}
\hline \multirow{8}{*}{$\frac{.00}{\frac{\pi}{\pi}}$} & & & & rsuc & & Sect & rd-est \\
\hline & & & I & II & III & IV & $\mathbf{V}$ \\
\hline & \multirow{5}{*}{ F1 } & Hauteur & +++ & ++ & + & ++ & +++ \\
\hline & & Exposition & +++ & ++ & ++ & ++ & +++ \\
\hline & & Formations quaternaires & $\mathrm{P}$ & $P$ & $\mathrm{P}$ & - & - \\
\hline & & Formations crétacés & $\mathrm{P}$ & $\mathrm{P}$ & - & $\mathrm{P}$ & $\mathrm{P}$ \\
\hline & & Tectonique (plissements + diaclases) & ++ & ++ & ++ & ++ & ++ \\
\hline & F2 & Présence & - & - & - & $\mathrm{P}$ & $\mathrm{P}$ \\
\hline \multirow{14}{*}{ 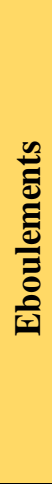 } & \multirow{6}{*}{ Paramètres } & Ls & ++ & + & ++ & + & +++ \\
\hline & & $\mathrm{Li}$ & ++ & + & ++ & + & +++ \\
\hline & & ls & ++ & + & +++ & + & ++ \\
\hline & & li & ++ & + & +++ & + & ++ \\
\hline & & $\mathrm{E}$ & +++ & + & ++ & + & ++ \\
\hline & & Di & ++ & + & ++ & + & +++ \\
\hline & \multirow{3}{*}{ Quantité } & Nombre des blocs & ++ & ++ & + & ++ & +++ \\
\hline & & Densité des blocs & ++ & + & + & +++ & +++ \\
\hline & & Volume des blocs & +++ & + & ++ & + & +++ \\
\hline & \multirow{3}{*}{ Indices } & $\mathrm{Ls} / \mathrm{Li}$ & + & + & + & + & + \\
\hline & & $\mathrm{Ls} / \mathrm{Di}$ & + & + & ++ & + & + \\
\hline & & $\mathrm{Ls} / \mathrm{E}$ & ++ & ++ & ++ & ++ & +++ \\
\hline & \multirow{2}{*}{ Disposition } & Direction de l'axe et inclinaison des blocs & ++ & ++ & ++ & ++ & ++ \\
\hline & & Direction des inclinaisons des blocs & + & ++ & +++ & + & ++ \\
\hline
\end{tabular}

Tableau 1: Tableau récapitulatif de différentes analyses effectuées (+++ fort, ++ moyen, + faible ; P présent, - absent).

A La surface, l'encroûtement accru, empêche le développement d'une végétation arbuste pouvant atténuer l'infiltration des eaux superficielles dans la falaise pouvant favoriser, en partie, son érosion.

De la lecture de l'évolution des valeurs des paramètres des blocs ressort que celles-ci sont variables et sont, relativement, influencées par la hauteur de la falaise (Tab. 1). En effet, la longueur et la diagonale ainsi que le contraste entre la longueur et la largeur des blocs sont également nettement élevés pour la zone $\mathrm{V}$ vis-à-vis des autres zones (surtout zones II et IV). Ceci peut être expliqué par le fait que, dans la zone V, la falaise est haute, ce qui permet aux diaclases de surface d'être moins touchées par les vagues et sont donc moins fragilisées, de même l'épaisseur des bancs massifs de calcaire est élevée dans cette zone, et par conséquent, les blocs se fragmentent peu lors de leur chute. Dans ce cas, leur longueur et leur diagonale restent intactes. Dans la zone I, la largeur et l'épaisseur des blocs, presque égales, montrent que ceux-ci ne sont pas longs et ont une section presque carrée. Ceci est dû à une fragmentation forte des blocs lors de leur chute, favorisée par la dureté de la plateforme (absence de sables) et les 
diaclases plus ou moins fragilisées par les vagues (falaise moins haute que dans la zone V).

Suite à ces constatations, on peut prétendre que les blocs, au pied de la falaise de Sidi Bouzid, héritent, partiellement, leur forme de la structure des formations qui composent cette falaise.

Concernant les valeurs du nombre et de la densité des blocs, elles sont variables, en général, et proportionnelles entre elles. Les plus élevées sont celles des zones I et $\mathrm{V}$ et témoignent que l'érosion à ces niveaux est très importante. Cette dernière est surtout favorisée, dans ces zones, par la hauteur importante et l'exposition de la falaise à la dynamique marine contrairement à ce qui se passe dans la zone III.

Quant aux valeurs du volume des blocs, elles évoluent de la même manière que celles de leur nombre et de leur densité à l'exception de la zone III (Tab. 1). Dans cette dernière, le nombre et la densité des blocs sont faibles alors que le volume des blocs est important. La cause étant la nature lithologique des blocs qui sont issus de l'érosion de dépôts de pente quaternaires très encroûtés et indurés mais moins affecté par les diaclases. Lors de leur chute, ils se fragmentent difficilement vis-à-vis des blocs issus de formations calcaires.

A propos des indices des blocs, Ls/Li, Ls/Di, Ls/E, ils montrent peu de variations surtout pour les indices faisant intervenir les grandes distances (Li et Di) (Tab. 1). Cependant, pour Ls/E qui fait intervenir l’épaisseur, la zone $\mathrm{V}$ se distingue des autres par son indice élevé (à cause d'une longueur très importante mais aussi très contrastée par rapport à l'épaisseur) et ce pour les mêmes arguments que ceux du paragraphe « paramètres des blocs ».

Pour ce qui est de la disposition des blocs exprimée par la direction de leurs axes, la direction de leur inclinaison et le degré de leur inclinaison, elle permet de mettre en évidence une légère différence entre les blocs du secteur nord-est et ceux du secteur sud-ouest en relation, en gros, avec l'orientation différente des falaises des deux secteurs produisant ces blocs (Tab. 1). Par contre, les valeurs très élevées de la direction de l'inclinaison des blocs dans la zone III s'expliquent, en plus de la direction de la falaise par la dérive littorale pour le secteur nord-est etpar la direction de la falaise ainsi que l'influence du virage, que forme cette zone par rapport aux autres, et au déferlement des vagues donnant naissance à un courant du NW au SE pour le secteur sud-ouest.

\section{Conclusion}

L'étude de la falaise avoisinant la belle plage marocaine de Sidi Bouzid montre que cette falaise côtière est complexe (F1 et F2), haute, discontinue et très exposée à la dynamique marine, ce qui justifie sa subdivision en deux secteurs et cinq zones. Sa géomorphologie prouve 
qu'elle garde encore les traces d'une influence des variations du niveau marin au cours du Quaternaire récent.

Ce travail, montre une nette relation étroite entre la quantité et la qualité des blocs, d'une part, et la nature du milieu de leur accumulation d'autre part.

Sur le plan stratigraphique, les falaises 1 et 2 sont formées, partout, d'une alternance de bancs de calcaires et de niveaux marneux crétacés qui sont surmontés, au Sud-Ouest, par des dépôts quaternaires essentiellement continentaux et encroûtés à la surface.

L'analyse des paramètres des blocs montre que leurs valeurs sont influencées par la hauteur de la falaise.La zone V, contrairement à la zone I, se différencie par des valeurs élevées de la longueur, de la diagonale et du contraste entre la longueur et la largeur de ses blocs suite à leur faible fragmentation lors de leur chute influencée par la hauteur élevée de la falaise, les diaclases à la surface moins fragilisées et l'épaisseur importante des bancs de calcaire. Ces constatations permettent de prétendre que les blocs, héritent, partiellement, leur forme de la morphologie, de la structure et de la nature lithologique des formations qui la composent.

L'analyse quantitative des blocs montre que leur nombre et leur densité sont variables et proportionnels mais plus élevés pour les zones I et V témoignant d'une érosion très active favorisée par la hauteur importante et l'exposition de la falaise à la dynamique marine. Le volume comme le nombre et la densité évoluent de la même manière à l'exception de la zone III où le nombre et la densité des blocs sont faibles alors que leur volume est important à cause de la nature lithologique des blocs issus de l'érosion de dépôts de pente quaternaires très encroûtés, indurés et riches en diaclases.

Concernant les indices des blocs, dépendant de Ls et Di, ils montrent peu de variations. La zone $\mathrm{V}$ se distingue des autres par l'indice de ses blocs Ls/E élevé pour les mêmes arguments cités dans le paragraphe " paramètres des blocs ».

Quant à la disposition des blocs, elle met en évidence une légère différence entre les blocs du secteur nord-est et ceux du secteur sud-ouest en relation avec l'orientation différente des falaises, dans les deux secteurs. Les valeurs très élevées de la direction de l'inclinaison des blocs dans la zone III s'expliquent, en plus de la direction de la falaise par l'influence du virage, que forme cette zone par rapport aux autres, et du déferlement des vagues donnant naissance à un courant NW au SE.

Dans ce travail, une variation spatio-temporelle de l'érosion qui affecte la falaise de Sidi Bouzid est démontrée. Elle a pour conséquence un recul, non négligeable de celle-ci, en présentant un risque qui exige une intervention pour la protection de cette falaise et des infrastructures avoisinantes (route, villas, restaurants et cimetière...). 


\section{References:}

ABERKAN M. (1996) - L’instabilité des formations quaternaires de la côte atlantique marocaine et son impact sur l'aménagement. 13ème Colloque des Bassins sédimentaires marocains. Marrakech, pp. 1.

ABERKAN M., ADIL S. et ZANNIBI F. (1997) - Le recul de la falaise côtière et le problème d'aménagement le long de la côte atlantique marocaine. 2ème Rencontre de l’Hydrodynamique marine. Ben M'SIK Casablanca.

AKIL M. (1990) - Les dépôts quaternaires littoraux entre Casablanca et Cap Beddouza (Méséta côtière marocaine) - Etudes géomorphologiques et sédimentologiques. Thèse Doc. Es. , Fac. Sci, Rabat, 417p.

ABOUMARIA Kh. (1993) - Les formations quaternaires du Sahel des Doukkala (Méséta occidentale marocaine) : mise en place et évolution postsédimentaire. Thèse de 3 ème cycle, Fac. Sci, Rabat, 186 p, 88 fig, 8 tab GIGOUT M. (1951) - Etude géologique sur la Méséta marocaine occidentale (arrière-pays de Casablanca, Mazagan et Safi). Trav. Inst. Sc. Chérifien, 3, et Not. Mém. Serv. Géol. Maroc, n 86, 2t. ,507 p.

KHATMI S.(1999) - La couverture méso-cénozoïque des Doukkala (Meséta côtière, Maroc) : Stratigraphie et évolution géodynamique. Doctorat 3éme cycle, El Jadida, 124pp.

OUADIA M.(1998) - Les formations plioquaternaires dans le domaine mésétien occidental entre Casablanca et Safi : Géomorphologie, Sédimentologie, Paléoenvironnements quaternaires et Evolution actuelle. Thèse Doct. d'Etat, Univ. Mohammed V, Rabat, 319 p.

OUADIA M., ABERKAN M. et ABOUMARIA K. (2015) - Le complexe dunaire quaternaire du Jorf Lasfar (Meseta côtière occidentale marocaine) : lithostratigraphie, sédimentologie et néotectonique. Paralia, Vol. 8, $\mathrm{N}^{\circ}$. 1, pp n02.1-n02.14, DOI: http://dx.doi.org/10.5150/revue-paralia.2015.n02.

SBAI F., LABRAIMI M. et HADDANE M. (2004) - « Evaluation du recul du trait de côte sur une portion du littoral atlantique marocain de Mohammedia» PANGEA 41/42 Juin / Décembre 2004.

TRICART J., JOLY F. et RAYNAL R. (1955) - Etude morphométrique des galets nord-africains. Notes Serv. géol. Maroc, t. 13, n 128, pp. 49-83. VOGT T. (1983) - Types et genèse de croûtes calcaires. Thèse Doc. Es. Sc., Fac. Sci, Paris, 253. 